\title{
CORRECTION
}

\section{BROWNIAN MODELS OF OPEN PROCESSING NETWORKS: CANONICAL REPRESENTATION OF WORKLOAD}

\author{
By J. MichaEL HARRISON
}

The Annals of Applied Probability (2000) 10 75-103

The paper above contains a substantial error, as shown by example in Section 7.1 of [2]. To correct this error one needs to add a restriction on the data of the model being analyzed, thus making explicit an assumption that is implicit in the original development. To enable a precise statement of that additional assumption, the next paragraph reviews some of the paper's notation and set-up.

Let $r, m$ and $n$ be positive integers, $R$ an $m \times n$ input-output matrix, $A$ a nonnegative $r \times n$ capacity consumption matrix, and $\lambda$ an $m$-vector of exogenous input rates. All of the paper's formal propositions relate, at least implicitly, to the following linear program, called the static planning problem (here displays are numbered exactly as in the original paper, for ease of reference): choose a scalar $\rho$ and an $n$-dimensional column vector $x$ of average activity rates so as to

$$
\begin{array}{ll}
\text { minimize } & \rho \\
\text { subject to } & R x=\lambda, \quad A x \leq \rho e \text { and } x \geq 0,
\end{array}
$$

where $e$ is the $m$-vector of ones. The dual of (2.1)-(2.2), or to be more precise, one formulation of its dual, is as follows: choose an $m$-dimensional row vector $\mu$ and $r$-dimensional row vector $\pi$ so as to

$$
\begin{array}{ll}
\operatorname{maximize} & \mu \lambda \\
\text { subject to } & \mu R \leq \pi A, \quad \pi e=1 \text { and } \pi \geq 0 .
\end{array}
$$

In the primal problem (2.1)-(2.2) one interprets $\lambda_{i}(i=1, \ldots, m)$ as the average rate at which a system manager is obliged to consume input $i$, or the average rate at which type $i$ input must be processed. On page 80 of the paper under discussion, the vector $\lambda$ is assumed to be nonnegative and nontrivial, but the nonnegativity is never actually used in the ensuing development; if $\lambda_{i}$ were negative one could interpret its absolute value as the average rate at which the system manager is obliged to supply material $i$.

To articulate the additional assumption referred to earlier, let us define the polyhedral cone

$$
V=\left\{v \in \mathbb{R}^{m}: R x=v \text { for some } x \geq 0\right\}
$$

Received September 2002. 
Obviously, then, the primal problem (2.1)-(2.2) is feasible (meaning that there exists a feasible solution $x$ ) if and only if $\lambda \in V$. Beyond this, however, the arguments presented in the paper implicitly assume that $\lambda$ lies in the interior of $V$. In the current context that requirement is conveniently expressed as follows.

Assumption 0. For each $\delta \in \mathbb{R}^{m}$ one has the following: $\left(\lambda+\frac{1}{t} \delta\right) \in V$ for all $t$ sufficiently large.

In linear programming language, this says that the primal problem (2.1)-(2.2) remains feasible for all sufficiently small perturbations of the vector $\lambda$. Of course, such a perturbation might have negative components if $\lambda_{i}=0$ for some $i$. This motivates use of the term exogenous flow rate in place of "exogenous input rate," so that exogenous inputs and exogenous outputs are treated symmetrically. One crucial implication of Assumption 0 is the following.

Proposition 0. The polyhedral set $\mathscr{D}$ of feasible solutions $(\mu, \pi)$ for the dual problem (2.3)-(2.4) has at least one extreme point.

Proof. Taking $\delta=0$ in Assumption 0, we have that $\lambda \in V$. Thus our primal problem (2.1)-(2.2) has a feasible solution, and because $\rho$ is bounded below by zero (remember that $A \geq 0$ ), it necessarily has an optimal solution. Thus the dual problem has an optimal solution as well, implying that $\mathscr{D}$ is nonempty. Then according to Theorem 2.6 on page 63 of [1], it suffices to show that $D$ does not contain a line. (The precise mathematical meaning of the phrase "D contains a line" is explained on that same page.) Now, making reference to the constraints (2.4) that define $\mathscr{D}$, let $\alpha$ be an $n$-dimensional row vector such that $\pi A \leq \alpha$ for all $\pi$ satisfying $\pi e=1$ and $\pi \geq 0$, and define the polyhedron $\mathcal{P}=\{\mu: \mu R \leq \alpha\}$. If $(\mu, \pi) \in \mathscr{D}$ then obviously $\mu \in \mathcal{P}$, so it suffices (because of the restrictions on $\pi$ in the dual problem) to show that $\mathcal{P}$ does not contain a line. Assumption 0 ensures that the rows of $R$ are linearly independent (i.e., $R$ has rank $m$ ), so $R$ also has $m$ columns that are linearly independent. The columns of $R$ are the coefficient vectors of the $n$ linear inequality constraints that define the nonempty polyhedron $\mathcal{P}$, so a second application of the Theorem 2.6 on page 63 of [1] establishes that $\mathcal{P}$ cannot contain a line.

The counter-example presented in Section 7.1 of [2] is one where $\mathscr{D}$ has no extreme points. Proposition 0 shows that Assumption 0 rules out such models. In its proof we have used only the fact that $R$ has rank $m$, but the full force of Assumption 0 is in fact needed later in the paper (specifically, in the proof of Proposition 2). That fact is somewhat obscured by the paper's expositional sequence, so we now describe an alternative sequence, including an alternative version of Proposition 1, that puts the relevant issues in sharper focus. In this alternative exposition the all-important Assumption 1 (the heavy traffic assumption) is imposed before Proposition 1 is stated. 
ASSUMPTION 1. The static planning problem (2.1)-(2.2) has a unique optimal solution $\left(\rho^{*}, x^{*}\right)$. Moreover, that solution has $\rho^{*}=1$ and $A x^{*}=e$.

Recall that we denote by $\left\{\left(\mu^{\ell}, \pi^{\ell}\right): \ell=1, \ldots, L\right\}$ the extreme points of the polyhedron $\mathscr{D}$. Equivalently stated, these pairs $\left(\mu^{\ell}, \pi^{\ell}\right)$ are the basic feasible solutions of the dual linear program (2.3)-(2.4). Also, the extreme points are numbered so that $\left\{\left(\mu^{\ell}, \pi^{\ell}\right): \ell=1, \ldots, L^{*}\right\}$ are the basic optimal solutions of (2.3)-(2.4). From Assumption 1 we then have the following:

$$
\mu^{\ell} \lambda=1 \quad \text { for } 1 \leq \ell \leq L^{*} \quad \text { and } \quad \mu^{\ell} \lambda<1 \quad \text { for } L^{*}<\ell \leq L .
$$

The alternative version of Proposition 1 referred to above is the following.

PROPOSITION 1. Let $\delta \in \mathbb{R}^{m}$ be arbitrary, let $t>0$ be large, and consider the linear programs (2.1)-(2.2) and (2.3)-(2.4) with $\lambda+\frac{1}{t} \delta$ in place of $\lambda$. For each sufficiently large $t$, each of these problems has an optimal solution and their (common) optimal objective value is

$$
1+\frac{1}{t} \max \left\{\mu^{\ell} \delta: \ell=1, \ldots, L^{*}\right\} .
$$

REMARK. This is precisely the property of the static planning problem that is invoked later in the proof of Proposition 2. Assumption 0 is obviously necessary for this property to hold.

Proof of Proposition 1. Exactly as in the proof of Proposition 0, Assumption 0 ensures that the primal problem (2.1)-(2.2) with $\lambda+\frac{1}{t} \delta$ in place of $\lambda$ has a feasible solution for all $t$ sufficiently large, and hence that it has an optimal solution for all such $t$. Thus the dual problem has an optimal solution as well, and because $\mathscr{D}$ has at least one extreme point by Proposition 0 (note that $\mathscr{D}$ does not depend on $\lambda$ ), there exists an optimal extreme point solution for the dual problem; cf. Theorem 2.7 on page 65 of [1]. That is, the optimal objective value is

$$
\max \left\{\mu^{\ell}\left(\lambda+\frac{1}{t} \delta\right): \ell=1, \ldots, L\right\} .
$$

Because we are interested only in large $t$, the desired conclusion is now immediate from (2.5).

All of the changes that have been described in this note occur in Section 2, and once they are made, the development in Sections 3 and beyond need not be altered in any way. In particular, Proposition 4 (in Section 5) is valid when the indicated changes have been made in Section 2. It concerns the "canonical representation of workload" referred to in the paper's title: as rows of the basis matrix $M$ one can take any maximal linearly independent subset of $\left\{\mu^{\ell}: \ell=1, \ldots, L^{*}\right\}$. 
If one imposes additional structural assumptions on the problem data $(R, A, \lambda)$, then Assumption 0 can be re-expressed in concrete, easily checkable terms. For example, conditions are presented in Section 7.2 of [2] which assure the validity of the "canonical representation" described above, and it is not difficult to verify that those conditions imply Assumption 0.

\section{REFERENCES}

[1] Bertsimas, D. and Tsitsiklis, J. N. (1997). Introduction to Linear Optimization. Athena Scientific, Belmont, MA.

[2] Bramson, M. and Williams, R. J. (2002). Two workload properties of Brownian networks. Unpublished manuscript.

GRADUATE SCHOOL OF BUSINESS

STANFORD UNIVERSITY

STANFORD, CALIFORNIA 94305-5015

E-MAIL: harrison_michael@gsb.stanford.edu 